\title{
El deterioro de la reputación de los profesionales de la comunicación tras la crisis del sistema mediático valenciano
}

\section{The decline in reputation of communication professionals following the crisis of Valencia's media system}
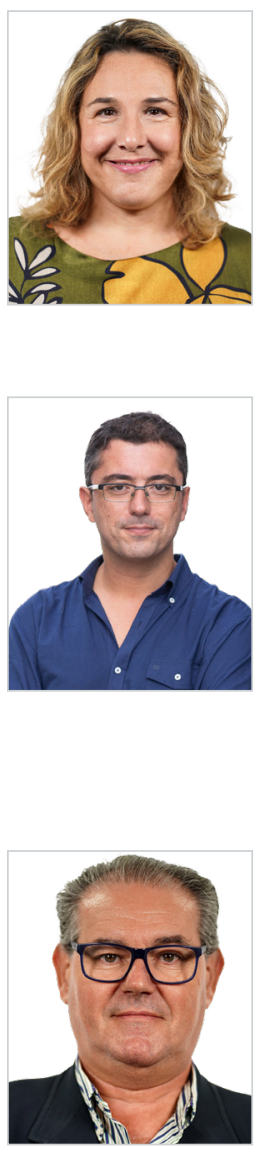

María Soler-Campillo. Licenciada en Ciencias Económicas y Empresariales por la Universitat de València, Máster en Asesoría Fiscal por el Instituto de Estudios Superiores del CEU-San Pablo de Valencia, Doctora en Comunicación Empresarial e Institucional por la Universitat Jaume I, es Profesora Contratada Doctora de Empresas de comunicación y Empresa audiovisual en la Universitat Jaume I. Ha publicado el libro Las empresas de fotografía ante la era digital. El caso de la Comunidad Valenciana (Madrid: Ediciones de las Ciencias Sociales, 2007), y participado en congresos, jornadas especializadas y publicado en revistas científicas sobre temas de economía y empresa audiovisual. Es miembro del Grupo de Investigación «Investigaciones en Tecnologías Aplicadas a la Comunicación Audiovisual» (ITACA-UJI).

Universitat Jaume I de Castellón, España

solerm@uji.es

ORCID: 0000-0002-3237-8038

Esteban Galán-Cubillo. Doctor en Comunicación y Contratado Doctor del Departamento de Comunicación, Audiovisual, Documentación e Historia del Arte de la Universidad Politécnica de Valencia, es Licenciado en Comunicación Audiovisual, Premio Nacional al mejor expediente académico. Ha desarrollado una larga trayectoria en producción y realización audiovisual, con más de 15 años de experiencia profesional en Canal 9, TVE y la Unión Europea. Su línea de investigación se centra en el estudio de las herramientas digitales en la producción audiovisual y en las narrativas transmedia, desde una perspectiva aplicada. Entre su producción científica destacan obras como Televisión en virtual (Madrid: IORTV, 2008) o Contenidos transmedia para la radiotelevisión de Proximidad (Pamplona: Eunsa, 2018). Es co-director del proyecto de investigación internacional From Ideas: Out of the box y es miembro del Grupo de Investigación ITACA-UJI. Desde 2017 dirige el podcast Transmedia y la plataforma de divulgación científica comtransmedia.com.

Universitat Politècnica de València, España

egalan@upv.es

ORCID: 0000-0001-8718-0937

Javier Marzal-Felici. Catedrático de Comunicación Audiovisual y Publicidad de la Universitat Jaume I de Castellón, es director del Departamento de Ciencias de la Comunicación y Coordinador del Programa de Doctorado. Es co-director de la Colección de libros "Guías para ver y analizar cine", co-director académico de la Colección Aldea Global que co-editan la Universitat Pompeu Fabra, la Universitat Autònoma de Barcelona, la Universitat Jaume I y la Universitat de València, y co-director de la Revista de periodicidad semestral adComunica. Revista sobre Estrategias, Tendencias e Innovación en Comunicación. Es Director del Grupo Investigaciones en Tecnologías Aplicadas a la Comunicación Audiovisual (ITACA-UJI), cuyos intereses se centran en el estudio de la cultura visual, las relaciones entre tecnología y visualidad, la teoría de la imagen, la economía política de la comunicación y el análisis de textos audiovisuales.

Universitat Jaume I de Castellón, España

marzal@uji.es

ORCID: 0000-0002-2462-1122

Cómo citar este artículo:

Soler-Campillo, M.; Galán-Cubillo, E. y Marzal-Felici, J. (2020). El deterioro de la reputación de los profesionales de la comunicación tras la crisis del sistema mediático valenciano. Doxa Comunicación, 31, pp. 41-61. 
Recibido: 02/05/2020 - Aceptado: 30/07/2020

\section{Resumen:}

En la última década hemos asistido al deterioro de la reputación de las profesiones vinculadas a los sectores de la comunicación y del audiovisual. Esta crisis reputacional se produce en plena recesión económica, tras el cierre de RTVV, la radiotelevisión pública de la Comunidad Valenciana entre 1989-2013. En este estudio se presentan los resultados de tres oleadas de cuestionarios realizados en 2015, 2017 y 2019 con el objetivo de conocer la percepción de los profesionales de los campos del periodismo, de la publicidad y del audiovisual sobre la reputación del propio sector comunicativo valenciano. Los resultados permiten constatar la existencia de una percepción negativa sobre la reputación de las profesiones relacionadas con la comunicación, que cabe relacionar con la debilidad del tejido asociativo y la ausencia de políticas de comunicación eficientes que favorezcan una interlocución efectiva con las administraciones públicas y con el conjunto de la sociedad valenciana.

\section{Palabras clave:}

Reputación; periodismo; publicidad; comunicación audiovisual; asociacionismo; Lobbying.
Received: 02/05/2020 - Accepted: 30/07/2020

\section{Abstract:}

Over the last decade, we have witnessed a decline in the reputation of professions linked to the communication and audio-visual sectors. This reputational crisis occurred in the midst of an economic recession after the closure of RTVV which was the public radio-television broadcaster of the Valencian Autonomous Region between 1989 and 2013. This study presents the results of three questionnaires carried out in 2015, 2017 and 2019 with the aim of gaining knowledge regarding the perception by professionals in the fields of journalism, advertising and audio-visual communication of the reputation of the Valencian communications sector itself. The results confirm a negative reputational perception of professions related to communication, which could be related to a weak structure of associations and the absence of efficient communication policies that might encourage effective dialogue with public administration and Valencian society as a whole.

Keywords:

Reputation; journalism; advertisement; audio-visual communication; promotion of associations; Lobbying.

\section{Introducción: algunos ejes para la reflexión}

\subsection{El valor de la reputación y los profesionales de la comunicación en la sociedad de la información}

Es bien sabido que el desarrollo de la sociedad de la información ha implicado y, al tiempo, ha sido consecuencia de la creciente relevancia de los medios de comunicación, que se ha visto acentuada con la aparición de los social media. En un mundo dominado por el poder de la imagen, la gestión de la reputación -de organizaciones, empresas, formaciones políticas, grupos sociales e, incluso, de individuos- se ha convertido en una herramienta fundamental para sobrevivir en el complejo ecosistema mediático contemporáneo. En una economía capitalista de carácter global, la imagen de las organizaciones es clave para su posicionamiento en el mercado, y para acumular uno de los valores más apreciados en la actualidad: la acumulación de capital reputacional (Teece, Pisana \& Shuen, 1997; Helfat \& Raubitschek, 2000; Costa, 2003).

En realidad, el concepto de reputación se asocia a un conjunto de valores como la «legitimidad», la «credibilidad», la «confianza» y la «transparencia» que inspira la correspondiente organización, institución, colectivo social o la persona física (Alloza, Carreras \& Carreras, 2013; Álvarez-Villanueva, 2012). En suma, la reputación es "uno de los recursos intangibles más valiosos de generación de valor y diferenciación de la empresa (...) es un activo estratégico para el mundo empresarial" (García-Santamaría, 2019: 201). En este sentido, existe un consenso bastante amplio entre los especialistas en reputación corporativa, a la hora de señalar que la imagen de las organizaciones es resultado de su comunicación, mientras 
que la reputación es consecuencia de la conducta de la organización, y de las percepciones que tengan sus diferentes públicos (stakeholders) (Villafañe, 2004, 2013). En las últimas décadas se han realizado grandes esfuerzos para desarrollar metodologías y técnicas de medición de la reputación de las corporaciones, entre cuyos rankings destacan el Hay Group Global Most Admired Companies, el Brandz ${ }^{\mathrm{TM}}$ Top 100 de Millward Brown, el RepTrak ${ }^{\mathrm{TM}}$ Pulse del Reputation Institute o el MERCO Empresas de Villafañe y Asociados, por citar sólo algunos pocos modelos (Villafañe, 2004; Álvarez-Villanueva, 2012; Alloza; Carrera; Carrera, 2013).

Con frecuencia se olvida que la propia actividad que desarrollan los comunicadores, generalmente organizados en asociaciones profesionales, también posee una dimensión reputacional. Desde hace décadas, en los principales foros de debate sobre la actividad de los comunicadores - periodistas, publicitarios y profesionales del audiovisual-se manifiesta el descontento por la insuficiente sensibilidad de la clase política, de muchas empresas e instituciones (como el sistema educativo), incluso de la sociedad en su conjunto, hacia la relevancia del papel de la comunicación.

Parece oportuno realizar una acotación preliminar. Cuando se habla de «sector comunicativo» o de «sectores de la comunicación", entendemos todas aquellas actividades relacionadas con la comunicación como el periodismo, la gestión de la comunicación empresarial e institucional o dirección de comunicación, la comunicación publicitaria, el entretenimiento y la ficción audiovisual, por lo que se van a considerar equivalentes, a efectos prácticos, las expresiones «sistema comunicativo» $\mathrm{y}$ «sistema audiovisual» $\mathrm{o}$ «sector de la comunicación» $\mathrm{y}$ «sector audiovisual» (CECUV, 2015). Por otra parte, es conveniente subrayar que las actividades profesionales relacionadas con la comunicación se enmarcan plenamente en el contexto de las industrias culturales y creativas, cuya relevancia para el conjunto de la economía ha sido ampliamente demostrada por numerosos estudiosos (Miége, 2004; Vogel, 2004; Castells, 2009; Zallo, 2011; Hesmondhalgh, 2012; Harvey, Wen \& Li, 2015; etc.) y por destacadas organizaciones como la UNESCO (2013).

En definitiva, la hipótesis que propone este artículo al estudiar la reputación del sector comunicativo y su relación con las condiciones laborales y la calidad del servicio que presta nos parece de gran importancia porque en la última década hemos sido testigos de un importante deterioro de la reputación de las profesiones vinculadas al campo de la comunicación, que ha desembocado en una precarización del sector y de las condiciones laborales de los profesionales. Por un lado, se ha podido constatar la pérdida de credibilidad del periodismo en los últimos años, como ha sido acreditado por diferentes informes de la Asociación de la Prensa de Madrid, en los informes anuales del Instituto Reuters para el estudio del Periodismo de la Universidad de Oxford y las encuestas periódicas del Centro de Investigaciones Sociológicas (APM, 2013, 2019; CIS, 2013, 2020; Valera, 2016; UPV, 2015; Newman et al., 2019).

En segundo lugar, si se presta atención a la situación del sector de la comunicación comercial y publicitaria, de la comunicación corporativa, de las agencias de medios y empresas de comunicación, se puede constatar que su evolución en la última década también ha sido negativa. Los Informes del Observatorio de la Publicidad en España, que publican la Asociación Española de Anunciantes (AEA), la Asociación de Creatividad Transformadora (ACT), la Asociación de Agencias de Medios (AM), el Consejo de Consumidores y Usuarios (AUC) y la Federación de Empresas de Publicidad y Comunicación, entre otros, permiten constatar una dramática disminución en inversión publicitaria entre 2007 y 2013, de 14.915 a 10.461 millones de euros, consecuencia de la profunda crisis financiera de 2008 (Benavides et al., 2015), datos avalados 
también por los Informes lnfoadex (Sánchez-Revilla et al., 2015). En efecto, en situaciones de crisis en países como España, donde el tejido económico es bastante débil, una de las primeras partidas que sufre recortes en cualquier empresa es la relacionada con la publicidad.

Por otro lado, destacan los resultados del European Communication Monitor de 2019, una macroencuesta realizada a cerca de 2.700 profesionales de la comunicación de 46 países europeos para conocer el prestigio y reputación de la profesión de comunicador, en especial de los directores de comunicación y gestores de relaciones públicas en empresas e instituciones. Este estudio subraya la necesidad de mejorar la transparencia en la gestión de los procesos internos y de los flujos de trabajo (Zerfass et al., 2019), y destaca la baja credibilidad que tienen los comunicadores y los periodistas (entre el $8 \%$ y el 17\%) en el conjunto de países de la UE.

Por otro lado, el sector audiovisual también ha sufrido de manera muy severa las consecuencias de la crisis económica de 2008. Las tradicionales ayudas a la producción cinematográfica y televisiva, tanto a nivel estatal como autonómico, han sufrido recortes drásticos en la última década, en especial desde 2012 (FAPAE, 2015). En el caso de la Comunidad Valenciana, los Informes de diferentes entidades como la Federación de Empresas Audiovisuales Valencianas (EAVF, 2014), la Mesa Sectorial del Audiovisual Valenciano (MESAV, 2014), de la Unió de Periodistes Valencians (UPV, 2015) o de la Comisión de Expertos en Comunicación de las Universidades Valencianas (CECUV, 2015) son muy elocuentes en lo referente a la grave situación del sector comunicativo y audiovisual valenciano, en especial en el periodo 2012-16, marcado por el cierre de la radiotelevisión pública valenciana en 2013 (Campos-Freire, 2013; EAVF, 2014; PAV, 2019).

\subsection{El colapso del sistema comunicativo: el caso de la Comunidad Valenciana como paradigma}

El impacto de la crisis financiera de 2008, así como los cambios en los modos de producción, distribución y consumo de los medios, además de la crisis de legitimidad de lo público, han tenido un fuerte impacto en el sistema comunicativo español y valenciano. El análisis de la evolución de la financiación de los servicios públicos audiovisuales permite constatar que la profundidad de la crisis ha sido mucho más acusada en los países del sur de Europa (Campos-Freire, 2016; Juanatey-Boga, Martínez-Fernández \& Rodríguez-Castro, 2018), que se ha recuperado levemente en el último trienio. No obstante, la Comunidad Valenciana constituye un caso de estudio realmente singular a nivel internacional y, como es evidente, en el contexto mediático español (Bustamante, 2015; Prado, 2015; Zallo, 2015; López-Rico, 2016a, 2016b). Sin duda, el cierre de RTVV en noviembre de 2013 ha marcado de forma dramática el desarrollo del sistema mediático valenciano.

En concreto, el 29 de noviembre de 2013 se produjo el cese de las emisiones de Ràdio 9 y de Canal 9, iniciándose así el proceso de liquidación de las dos sociedades públicas de radiotelevisión dependientes de la Generalitat Valenciana, RTVV S.A. (Televisió Autonòmica Valenciana S.A. y Ràdio Autonomia Valenciana S.A.). En 2015 se produce la entrada de un nuevo gobierno sustentado en partidos de izquierdas tras veinte años de gobiernos conservadores. Ante un escenario mediático valenciano devastado, en el que la Comunidad Valenciana pasa a convertirse en el único territorio de la Unión Europea, con lengua y cultura propias, privado de servicio público de radiotelevisión, el nuevo gobierno surgido de las elecciones de 2015 inició el proceso para recuperar los medios de comunicación públicos valencianos. Tras muchos meses de trabajo y presiones por parte de las asociaciones profesionales del sector comunicativo valenciano, a través de 
la Mesa Sectorial del Audiovisual Valenciano (MESAV), que agrupaba a trabajadores, empresarios y académicos de las universidades valencianas, y la complicidad del nuevo gobierno, se consiguió la aprobación por las Cortes Valencianas de la Ley 6/2016, de 15 de julio del Servicio Público de Radiodifusión y Televisión de ámbito autonómico, de titularidad de la Generalitat.

La recuperación del servicio público de RTV en la Comunidad Valenciana ha sido un proceso complejo, producido además en un contexto político muy agitado, con una audiencia atomizada y con hábitos de consumo multipantalla que poco tienen que ver con el escenario mediático de 2013, cuando se produjo el cese de emisiones (Galán Cubillo, Gil-Soldevilla, Soler-Campillo \& Marzal-Felici, 2018). Pero también, con una ciudadanía muy distanciada de RTVV, desafección que no ha desaparecido durante estos años. Hay que subrayar que, en el periodo 2012-2020, la prensa nacional y regional ha realizado un seguimiento del proceso de cierre de RTVV poco riguroso, incluso del proceso de creación de À Punt Mèdia, cercano con frecuencia al sensacionalismo, llegando a «adquirir dimensiones propias de un auténtico 'culebrón'», por ejemplo, con los despidos de miles de profesionales o el tema de las baremaciones de las bolsas de trabajo para la contratación de la nueva plantilla de APM, que vino a satisfacer la curiosidad (morbosa) de una audiencia muy desinformada e intoxicada por el constante «ruido informativo» sobre el caso, donde ha sido frecuente la confusión entre «información» y «opinión» (Marzal-Felici \& Soler-Campillo, 2017). En definitiva, la crisis de RTVV ha empeorado la reputación de las profesiones relacionadas con la comunicación y con el audiovisual. Y es evidente que los propios comunicadores han tenido y tienen una gran responsabilidad en la precaria reputación que posee la comunicación como actividad en la sociedad valenciana (y española).

\section{Metodología}

El estudio que presentamos combina diferentes técnicas de investigación: revisión bibliográfica y documental sobre la temática objeto de estudio; análisis PEST, esto es, del entorno general -de los factores Políticos, Económicos, Sociales y Tecnológicos- que constituyen el marco contextual del sistema comunicativo español y valenciano; técnica de observación participante, dado que los autores de este trabajo forman parte y han participado en las actividades de algunas organizaciones profesionales objeto de análisis; realización de encuestas con preguntas cerradas basadas en escalas de likert sobre la imagen reputacional del sector comunicativo valenciano; y realización de entrevistas en profundidad a dirigentes y profesionales relevantes del sistema comunicativo y audiovisual valenciano.

\subsection{El diseño de los cuestionarios}

El diseño de las preguntas ha seguido el modelo de cuestionario basado en la escala psicométrica de Likert, con el fin de objetivar el acuerdo o desacuerdo sobre una afirmación, mediante una escala ordenada y unidimensional de 1 a 5, a la que se ha incluido una opción de ausencia de opinión, que permite mejorar la calidad de los datos a obtener (Matas, 2018). Por otra parte, los autores del presente trabajo han seguido también la técnica de observación participante (Duverger, 1971), puesto que en los últimos años han participado en numerosas actividades desarrolladas «en la vida del grupo u organización que se estudia» (Piñeiro-Aguilar, 2015: 82), dando lugar a la creación de un diario de campo, donde 
se describía y analizaban los resultados obtenidos, además de participar en la redacción de varios informes técnicos, como el Informe CECUV (2015), o como expertos invitados en diferentes Comisiones de las Cortes Valencianas que han trabajado en la reactivación del sector audiovisual valenciano. De manera complementaria, también se han realizado una decena de entrevistas no estructuradas a los principales responsables de las asociaciones profesionales, al participar en organizaciones como la MESAV (Mesa del Audiovisual Valenciano), la CECUV (Comisión de Expertos en Comunicación de las Universidades Valencianas) o la CIRTPV (Comisión Interuniversitaria en favor de una Radiotelevisión Pública Valenciana) entre 2013 y 2015, cuyos resultados sirvieron para elaborar los cuestionarios que a continuación se describen.

Las encuestas de 2015, 2017 y 2019 han sido realizadas con la ayuda de la herramienta «Formularios de Google», y los enlaces a los formularios fueron distribuidos a los profesionales de la comunicación con la colaboración de los responsables de las organizaciones que, desde marzo de 2014 hasta finales de 2019, han formado parte de la Mesa Sectorial del Audiovisual Valenciano (MESAV), espacio de debate que carece de estatuto legal (puesto que no es una asociación profesional, ni una fundación sin ánimo de lucro). En la tabla no 1 , se reproduce una relación de asociaciones profesionales y de instituciones que forman parte del universo de destinatarios del cuestionario. A falta de censos exactos de carácter público, se estima que el universo total de encuestados podría situarse en torno a 2.500 profesionales. Las cifras sobre el número de miembros de cada organización es aproximado, puesto que no existen datos oficiales ni censos exactos, de carácter público, por lo que se han obtenido a través del contacto directo con sus responsables y/o con la información disponible en sus páginas web. Puede ocurrir que algunos profesionales tienen vinculación con varias organizaciones al mismo tiempo.

Tabla no 1 . Asociaciones, organizaciones y colectivos profesionales destinatarios de las encuestas de 2015, 2017 y 2019

\begin{tabular}{|l|c|}
\hline \multicolumn{1}{|c|}{ Organización } & Número \\
\hline Actors i Actrius Professionals Valencians (AAPV) & 30 \\
\hline Asociación de Directivos de Comunicación en la Comunitat Valenciana y Región de Murcia & 35 \\
\hline Asociación para el Desarrollo de la Comunicació (adComunica) & 110 \\
\hline Asociación de la Prensa de Alicante (APA) & 280 \\
\hline Associació Ciutadania i Comunicació (ACICOM) & 25 \\
\hline Associació de Dones Cineastes i de Mitjans Audiovisuals (CIMA) & 10 \\
\hline Associació de Compositors de Música per a l'Audiovisual & 15 \\
\hline Associació d'Empreses de Serveis de l'Audiovisual Valencià (AESAV) & 40 \\
\hline Associació de Productors Audiovisuals Valencians (PAV) & 100 \\
\hline Associació de Fotògrafs Professionals de València (AFPV) & 250 \\
\hline Associació de Treballadors de l'Audiovisual Valencià (ATRAU) & 174 \\
\hline Associació per a la Recuperació de la Memòria Internacional Audiovisual (APREMIA) & 2 \\
\hline Associació Valenciana d'Empreses de Producció Audiovisual i Productors Independents (AVANT) & 25 \\
\hline Associació Valenciana d'Empreses Productores d'Animació (AVEPA) & 15 \\
\hline Associació Valenciana d'Estudis d'Animació (ANIMAT) & 15 \\
\hline Associació Valenciana d'Estudis de Doblatge i Sonorització (AVEDIS) & 5 \\
\hline Associació Valenciana de Productors Independents (AVAPI) & 20 \\
\hline Colegio Oficial de Publicitarios y Relaciones Públicas de la Comunidad Valenciana & 180 \\
\hline
\end{tabular}




\begin{tabular}{|l|c|}
\hline Col.lectiu de Professionals del Doblatge (CPD) & 15 \\
\hline Empreses de Comunicació Publicitària de la Comunitat Valenciana (ComunitAD) & 50 \\
\hline Escriptors de l'Audiovisual Valencià (EDAV) & 120 \\
\hline Productors, Directors, Guionistes, Associats (PRODIGA) & 5 \\
\hline Unió de Periodistes Valencians (UPV) & 700 \\
\hline Acadèmia Valenciana de l'Audiovisual (AVAV) & 45 \\
\hline Departament de Ciències de la Comunicació. Universitat Jaume I & 67 \\
\hline Departament de Comunicació Audiovisual, Documentación e Historia del Arte. Universitat Politècnica de València & 45 \\
\hline Departament de Teoria dels Llenguatges i Ciències de la Comunicació. Universitat de València & 57 \\
\hline Departament de Comunicació i Psicologia Social. Universitat d'Alacant & 78 \\
\hline Departamento de Ciencias Sociales y Humanas. Universitat Miguel Hernández d'Elx & 38 \\
\hline Departamento de Comunicación Audiovisual y Publicidad. Universidad Cardenal Herrera CEU & 33 \\
\hline Departamento de Comunicación e Información Periodística. Universidad Cardenal Herrera CEU & 13 \\
\hline TOTAL & 2.597 \\
\hline
\end{tabular}

Elaboración propia

\subsection{Preguntas de investigación}

En primer lugar, cabe señalar que, aunque el concepto de reputación ha sido utilizado sobre todo en el análisis de los bienes intangibles de corporaciones u organizaciones empresariales y, asimismo, para explicar la construcción de la marca personal y el desarrollo del liderazgo (político, social, organizacional, etc), se considera que la teoría de la reputación puede y debe ser aplicada al estudio y análisis de la actividad profesional del propio sector comunicativo y audiovisual (Alloza, Carreras \& Carreras, 2013; Ortiz, 2015; Villafañe, 2013).

Por una parte y tras analizar los resultados obtenidos del trabajo de observación participante desarrollado entre 2013 y 2017 a través de la participación en diferentes foros y comisiones de expertos para la reapertura del servicio público de radiotelevisión y del análisis de los informes y publicaciones especializados, se ha estimado imprescindible conocer la percepción de los profesionales sobre el seguimiento del cierre de RTVV, un hecho que ha tenido una enorme repercusión sobre la reputación de los profesionales de la comunicación a nivel social, y que concretamos en una serie de preguntas en las encuestas de 2015 y 2017 :

1. ¿Qué percepción tienen los profesionales de la comunicación valencianos sobre los medios de comunicación y, en particular, sobre el tratamiento informativo del cierre de RTVV?

2. ¿Qué enfoques han adoptado las cabeceras en papel y digitales a la hora de informar sobre el seguimiento del cierre de RTVV?

3. ¿Cómo ha sido el seguimiento informativo sobre la repercusión internacional del cierre de RTVV?

Estas preguntas son el resultado del análisis de la información obtenida a través de las entrevistas con los responsables de las diferentes asociaciones y de la observación participante. De ellas, se extrajo la necesidad de estudiar el seguimiento que el proceso de cierre de RTVV había tenido por parte del resto de medios y sobre la reputación de los profesionales de 
la comunicación. Por otra parte, se consideró oportuno conocer la percepción de los profesionales de la comunicación sobre la reputación del sector comunicativo y audiovisual valenciano, casi un año después de la puesta en marcha de À Punt Mèdia, asunto que se aborda en la encuesta de 2019:

4. ¿Cómo perciben los profesionales valencianos el reconocimiento social de la comunicación en la Comunidad Valenciana, en España y en comparación con los países más avanzados de la OCDE?

5. ¿Se considera que los medios de comunicación han empeorado o mejorado el prestigio de las profesiones vinculadas a la comunicación?

6. ¿Cómo se valora el papel de la MESAV, la campaña de comunicación de la MESAV "Voces para un nuevo espacio audiovisual" o la creación de la Academia Valenciana del Audiovisual"?

En definitiva, la realización de las encuestas ha tenido como objeto valorar la situación reputacional del sector comunicativo y audiovisual y las preguntas se han adaptado a la situación cambiante del sector durante los seis años en los que ha transcurrido esta investigación. En las oleadas del 2015 y 2017 se mantuvo la misma estructura, y en 2019 se decidió introducir las cuestiones 4, 5 y 6 para poder monitorizar los cambios que había experimentado el sector.

\section{Resultados}

\subsection{La baja participación en las encuestas: un primer diagnóstico}

Uno de los aspectos más llamativos de las tres oleadas de encuestas que hemos realizado en 2015, 2017 y 2019 ha sido la escasa participación de los profesionales de la comunicación -con 111, 111 y 93 respuestas, respectivamente-, sobre un universo de 2.500 potenciales encuestados.

Gráfico nº 01. Número de encuestados en las tres oleadas

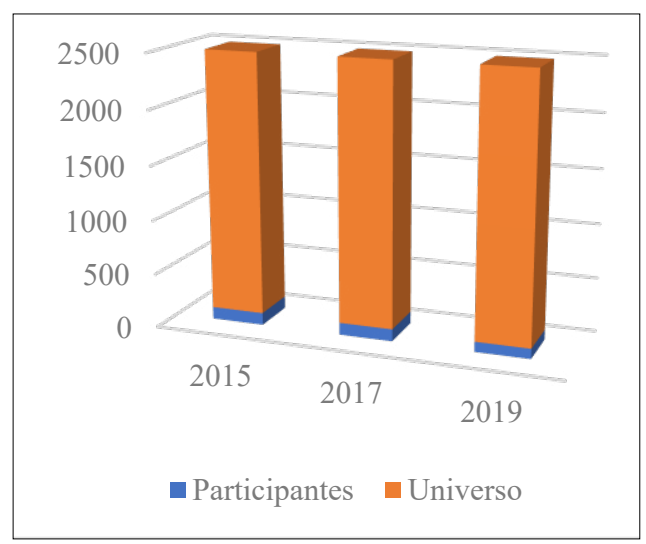

Elaboración propia 
Por lo que respecta a la distribución por sexos, se constata una mayor participación de hombres que de mujeres.

\section{Gráfico no 02. Distribución por sexos de los encuestados}

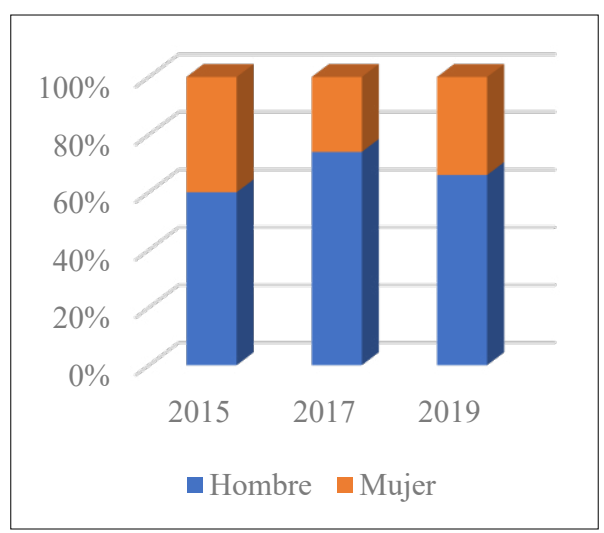

Elaboración propia

La baja participación de los profesionales de la comunicación en las encuestas se puede relacionar con el contexto PEST, con el escenario de crisis que ha vivido el sistema comunicativo valenciano. En este sentido, se dispone de información sobre la situación laboral de los que encuestados, que guarda relación con la evolución de los sectores de la comunicación entre 2015-2019.

\section{Gráfico n 03. Situación laboral de los encuestados en las tres oleadas}

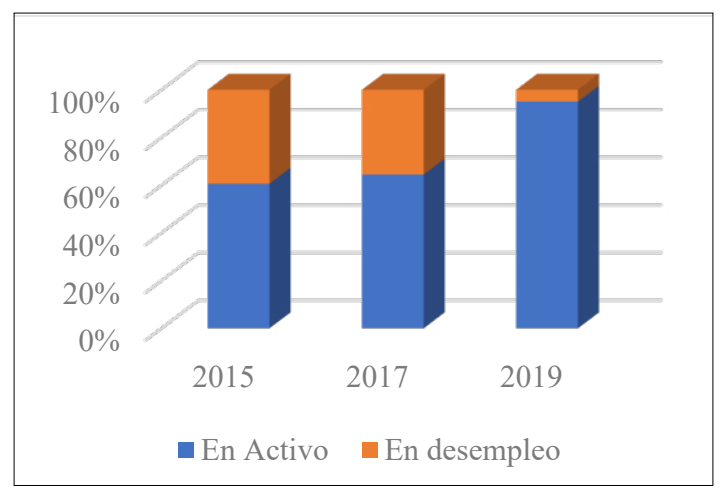

Elaboración propia

Otro aspecto interesante se refiere a la identificación del ámbito profesional de los encuestados, que ha permanecido bastante estable en las tres encuestas, destacando la participación mayoritaria de periodistas y profesionales del audiovisual, 
la muy escasa participación de los profesionales del campo de la comunicación publicitaria y una tímida participación del mundo académico, en torno al $17 \%$ del total, muy reducida si se tiene en cuenta que el número de académicos del campo de la comunicación en la CV es cercano a 330 profesores universitarios, entre las 6 universidades valencianas con estudios de comunicación. En la encuesta de 2019 destaca el aumento de la participación del colectivo de académicos, con respecto a otros colectivos.

\section{Gráfico no 04. Identificación del ámbito profesional de los encuestados en las tres oleadas}

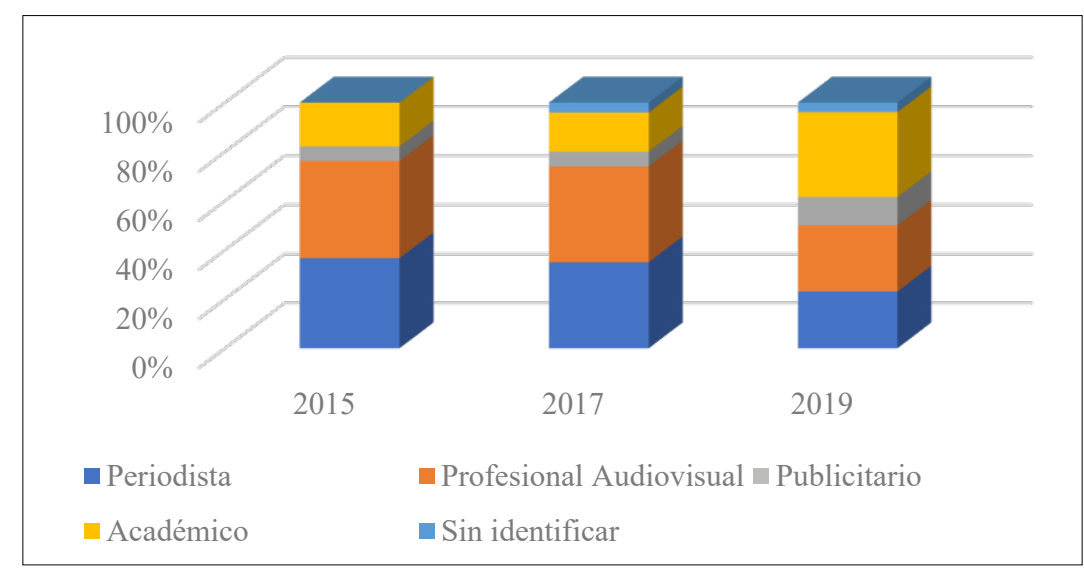

Elaboración propia

En suma, se considera que la escasa respuesta de los profesionales de la comunicación a las sucesivas encuestas es, asimismo, indicativa de la falta de conciencia del propio sector comunicativo sobre la relevancia de la comunicación en la sociedad que vivimos, un asunto que nos parece de notable gravedad: la falta de cultura corporativa es manifiesta entre los propios comunicadores.

\subsection{El tratamiento informativo del cierre de RTVV en las encuestas de 2015 y 2017}

El presente estudio se diseña a principios de 2015, coincidiendo con el cambio de gobierno de la Generalitat Valenciana, con la promesa de restituir el servicio público de radiotelevisión. En julio de 2015, se realiza la primera encuesta online, centrada especialmente en la percepción del seguimiento informativo del proceso de cierre de RTVV por parte de los principales periódicos en papel y digitales de España y de la Comunidad Valenciana, dirigida a los diferentes colectivos que integran la Mesa Sectorial del Audiovisual Valenciano (MESAV). La encuesta se volvió a realizar en 2017 con los siguientes resultados.

El 96\% de los encuestados, tanto en 2015 como en 2017, manifiestan haber seguido el proceso de cierre de RTVV a través de la prensa analógica y digital desde el anuncio de su cierre, y un 40\% se declaraban como informadores de dicho proceso. 
A la pregunta sobre si los medios de comunicación han realizado un seguimiento riguroso del proceso de cierre de RTVV, existe una coincidencia casi absoluta en los resultados de las encuestas en 2015 y 2017: el 18-19\% lo califican de "nada riguroso", el 36-35\% de los encuestados lo consideran poco riguroso, mientras que un 34-37\% tiene una opinión neutral, un 11-12\% señala que la prensa ha realizado un seguimiento bastante riguroso y sólo 1-1\% que ha sido riguroso.

Gráfico no 05. Consideración porcentual sobre el rigor informativo en el seguimiento del cierre de RTVV

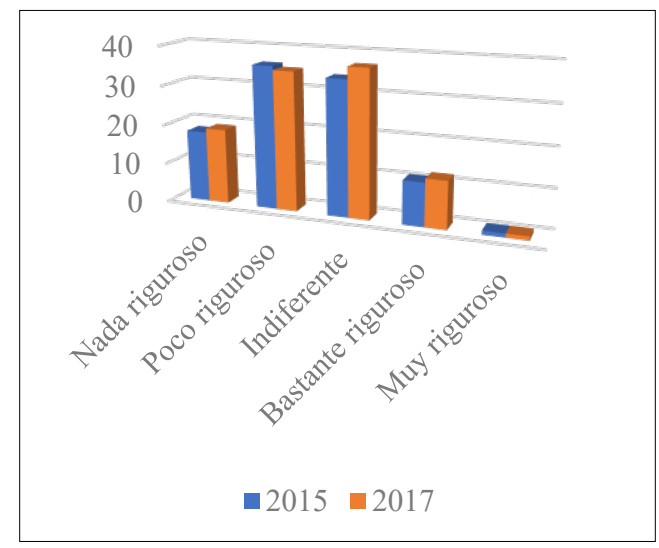

Elaboración propia

Los cuestionarios de 2015 y 2017 incluyeron preguntas acerca de los enfoques adoptados por la prensa escrita y digital a la hora de la informar sobre el seguimiento del cierre, destacando los siguientes argumentos:

- Se ha prestado mucha atención al debate político que existe en el trasfondo del cierre de RTVV: alrededor del 50\% de los encuestados lo consideran relevante o muy relevante en las encuestas de 2015 y 2017.

- El tratamiento del cierre de RTVV por parte de la prensa ha ignorado la problemática de los trabajadores de RTVV. Alrededor del 50\% de los encuestados en 2015 y 2017 consideran que este enfoque ha sido poco o nada relevante.

- Alrededor del 70\% de los encuestados en 2015 y 2017 señalan que el enfoque de las noticias sobre el cierre de RTVV ha ignorado su impacto en las industrias culturales creativas valencianas así como la problemática de la defensa de la lengua y la cultura valencianas.

- Finalmente, también hay una amplia coincidencia a la hora de valorar de forma negativa el tratamiento del proceso de cierre de RTVV. Cerca dos tercios de los encuestados en 2015 y 2017 consideran que el enfoque ha sido sensacionalista.

A continuación, el cuestionario recoge la valoración sobre la calidad del seguimiento informativo del cierre de RTVV en las cabeceras más influyentes en la comunidad. De manera general, los encuestados consideran que el seguimiento del cierre de RTVV ha sido más completo en los medios digitales (53\%, en 2015; 56\%, en 2017) frente a los medios impresos (15\%, en 2015 y 2017), si bien un tercio consideran que el tratamiento ha sido similar (32\%, en 2015; 35\%, en 2017). 
Tanto en el cuestionario de 2015 como en el 2017 se plantea una pregunta sobre las razones que explican la escasez de noticias, artículos de opinión y editoriales sobre el proceso de cierre de RTVV en la prensa española y valenciana, con 5 respuestas posibles, de las que se podían seleccionar dos:

- El 51\% (55\% en 2017) de las respuestas consideran que la razón es «porque se trata de un tema muy politizado, que resulta incómodo para muchos periodistas».

- El 47\% (51\% en 2017) han marcado la opción «Por intereses económicos, ya que la desaparición de RTVV supone un competidor menos en el escenario mediático».

- El 43\% (46\% en 2017) de los encuestados han seleccionado la opción «Porque buena parte de la profesión periodística no tiene conciencia de la importancia de que existan radiotelevisiones públicas de proximidad».

- El 13\% (15\% en 2017) seleccionó la opción «Porque el tema del cierre de RTV no tiene interés para una mayoría de ciudadanos».

A continuación, se formularon preguntas sobre la repercusión internacional del cierre de RTVV. La primera pregunta (de respuesta simple Sí / No / No conozco la noticia) sobre el tratamiento informativo de la denuncia del cierre de RTVV ante Naciones Unidas por la ONG noruega “Global Network for Rights and Development” y por el "International Institute for Peace, Justice and Human Rights” en la prensa en papel y/o digital, que el 57\% (el 56\% en 2017) valora de forma negativa, mientras el 43\% (el 44\% en 2017) manifiesta simplemente desconocer. La segunda pregunta planteaba si la noticia de la queja presentada por Izquierda Unida del País Valenciano ante la Comisión de Peticiones del Parlamento Europeo por el cierre de RTVV, entendida como violación de la Carta Europea de las Lenguas Minoritarias y del Tratado de Amsterdam, ha recibido un tratamiento informativo adecuado en la prensa impresa y/o digital (de respuesta simple Sí / No / No conozco la noticia). El 75\% de los encuestados manifiestan que el tratamiento fue inadecuado, mientras que el $20 \%$ declaran desconocer esa iniciativa, con idéntico resultado en las encuestas de 2015 y 2017.

Finalmente, el cuestionario habilita una pregunta abierta para que los encuestados pudieran incluir sus comentarios y reflexiones sobre la problemática planteada, lo que ha sido de gran utilidad para identificar las inquietudes y valoraciones de los encuestados acerca de la situación del sector comunicativo y audiovisual español y valenciano.

\subsection{Resultados de la encuesta de 2019}

Finalmente, en abril de 2019 se realizó una nueva encuesta, en esta ocasión incorporando aquellos aspectos relativos a la problemática de la reputación del sistema comunicativo valenciano que se han modificado durante estos años. La encuesta fue dirigida a los mismos colectivos profesionales y sirve también para comparar los resultados de 2015 y 2017 , cuando en 2019 ya se había cumplido más de un año de existencia de los nuevos medios de comunicación públicos valencianos, que constituyen un «motor» esencial para el desarrollo del sistema mediático público-privado y para el desarrollo de las industrias culturales valencianas (Prado, 2015; Marzal-Felici \& Soler-Campillo, 2015). En esta ocasión, como hemos señalado, se han obtenido 93 respuestas. 
- El 59\% de los encuestados señala que la comunicación, entendida de una manera amplia -es decir, englobando todo aquello que tiene que ver con la información, el entretenimiento y la comunicación publicitaria e institucional-, goza de un mediocre reconocimiento social en la Comunidad Valenciana.

- El 38\% considera que esta situación es más negativa en la Comunidad Valenciana que en el resto de España.

- El 73\% coincide en señalar que en los países más avanzados de la OCDE (como Estados Unidos, Canadá, Francia, Reino Unido, Alemania, Japón, Italia, Holanda, Dinamarca, etc.) existe una conciencia más clara sobre la relevancia de la comunicación como herramienta para el progreso económico, el bienestar social y la salud democrática que en España.

- Cerca del $82 \%$ considera, de manera negativa o muy negativa, que los medios de comunicación, de cobertura estatal, han ayudado a empeorar el prestigio de las profesiones relacionadas con la comunicación (actividades profesionales como periodista, publicitario, guionista, realizador, productor, director de comunicación, etc.).

- A la hora de valorar si la recuperación de los medios de comunicación públicos valencianos, À Punt Mèdia, ha contribuido a mejorar la reputación de las profesiones relacionadas con la comunicación y el audiovisual valenciano, el $44 \%$ considera que nada o poco.

- Casi el $82 \%$ de los encuestados valora negativa o muy negativamente que los ámbitos profesionales de la comunicación -de la información, de la comunicación publicitaria e institucional y del entretenimiento- dependan tan estrechamente de la actividad política

De este modo, se constata que entre los profesionales de la comunicación de la Comunidad Valenciana existe una percepción bastante negativa sobre la reputación de las profesiones vinculadas a la comunicación, a diferencia de lo que ocurre en los países más avanzados, situación que no mejoran los medios de comunicación estatales o locales, ni siquiera la recién creada À Punt Mèdia, la sociedad mercantil de la nueva Corporación Valenciana de Medios de Comunicación (CVMC).

\subsubsection{Análisis de las iniciativas de mejora de la reputación del sector comunicativo: MESAV y AVAV}

En esta encuesta de 2019 se pregunta sobre el papel desempeñado por la Mesa Sectorial del Audiovisual Valenciano MESAV (creada en 2014 tras el cierre de RTVV) y por la Academia Valenciana del Audiovisual creada en 2018. Con respecto al papel jugado por la MESAV en el proceso de recuperación del audiovisual valenciano, valorándose principalmente tres aspectos:

- Para el 50\% de los encuestados, la MESAV ha sido muy útil como espacio para poner en común los problemas que comparten los profesionales de la comunicación.

- Para el 44\% de los encuestados, la puesta en marcha de la MESAV ha sido importante como ejercicio de análisis y de crítica constructiva de las políticas de apoyo a los sectores de la comunicación.

- Finalmente, el 39\% de los encuestados coinciden en señalar que la MESAV ha sido relevante como ejercicio de transparencia ante la propia profesión y ante la sociedad. 
No obstante, resulta llamativo que para el $71 \%$ de los encuestados, la MESAV no ha sido un instrumento útil para ganar visibilidad ante la sociedad valenciana, el 65\% considera que la MESAV no ha servido para demostrar la unidad de todos los sectores de la comunicación ante la sociedad valenciana y para el 72\% de los encuestados la MESAV no ha servido tampoco para comunicar a la sociedad los problemas del sector audiovisual y de la comunicación valencianos.

De este modo, se puede afirmar que una parte importante de la profesión de comunicador no ha entendido el papel jugado por la MESAV en estos últimos años. Como participantes en este espacio de debate, podemos señalar que ha existido una falta de implicación de buena parte de las asociaciones profesionales, que cabe relacionar con la ausencia de cultura corporativa sobre la realidad de la comunicación, en la que prevalece una visión estrecha y cortoplacista sobre la propia profesión.

En lo referente a la Academia Valenciana del Audiovisual Valenciano (AVAV) conviene señalar que es una asociación que nace con el objetivo de trabajar para la mejora de la reputación del sector y de las profesiones relacionadas con el audiovisual valenciano, para lo que ha contado con el apoyo del Instituto Valenciano de Cultura, Sección Audiovisual y Cinematografía, en especial para la creación de los Premios del Audiovisual Valenciano.

En la encuesta de 2019 se incluye una pregunta acerca de la creación de la "Acadèmia Valenciana de l'Audiovisual", nacida en julio de 2018, sobre su relevancia para potenciar el audiovisual valenciano y para mejorar la reputación de las profesiones relacionadas con el sector audiovisual.

Gráfico no 06. Respuesta de los encuestados en 2019 sobre la creación de la Academia Valenciana de l'Audiovisual

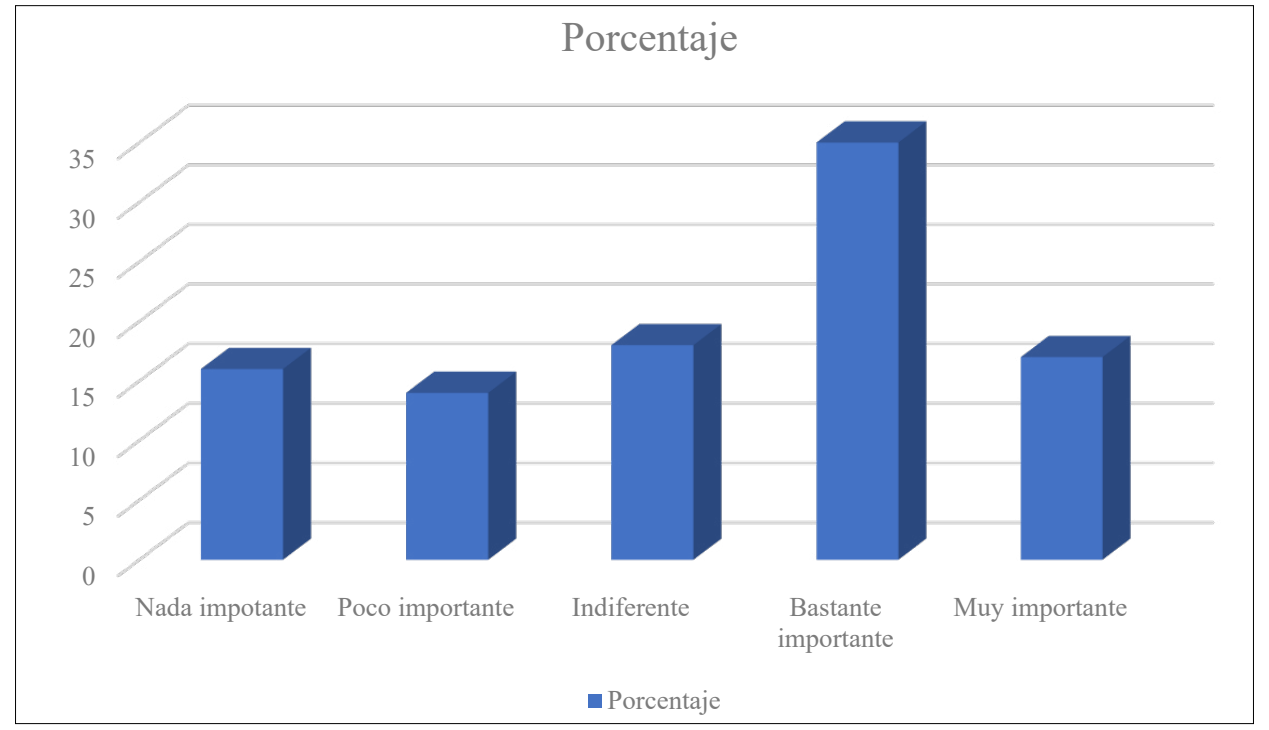

Elaboración propia 
Como se puede constatar en el gráfico, más del $52 \%$ considera que la aparición de la AVAV es bastante o muy importante. De los 60 miembros con que la AVAV contaba inicialmente se ha pasado a 145 en poco más de año y medio, lo que indica la existencia de una voluntad colectiva para crear una gran plataforma que aglutine los intereses del sector audiovisual, si bien en este espacio no tienen cabida los profesionales de la información y de la comunicación publicitaria que sí han sido incorporados en los cuestionarios realizados en este trabajo.

\section{Discusión y conclusiones}

Por un lado, el análisis de las respuestas a las encuestas de 2015 y 2017 permiten constatar que el tratamiento informativo del cierre de RTVV, incluso de su repercusión a nivel internacional -por las denuncias ante Naciones Unidas o el Parlamento Europeo- ha sido percibido por los profesionales de la comunicación de forma bastante negativa, por tratarse de un tema politizado, en el que interfieren los intereses económicos de los medios privados y que indica que numerosos periodistas no entienden el papel que juegan los medios públicos en un sistema mediático moderno. No obstante, una quinta parte de los encuestados desconocía la existencia de las denuncias internacionales. Los resultados de estas encuestas vienen a confirmar que en el seguimiento del cierre de RTVV ha habido una manifiesta falta de imparcialidad y rigor en buena parte de la prensa, como se puede constatar en un análisis crítico de las noticias publicadas en ese periodo (Marzal-Felici \& Soler-Campillo, 2017).

Con respecto a la cuestión reputacional, los resultados del presente trabajo reflejan la necesidad que tienen los profesionales de la comunicación de mejorar la imagen que la profesión de comunicador tiene acerca de su propio colectivo profesional. Este ejercicio de «autoconocimiento», necesariamente autocrítico, es esencial para entender las causas que explican la escasa relevancia que tiene la comunicación, no sólo en los contextos profesionales próximos a la comunicación, sino también para conocer las causas que explican la desafección y falta de valoración de la importancia de la comunicación para la sociedad. En el ámbito del periodismo, "la mejor receta para paliar los efectos de la crisis del sector y revertir la pérdida de credibilidad, reputación e intermediación (...) transita por el camino de la excelencia periodística, la cual comporta la calidad informativa y el cumplimiento de los más altos estándares éticos y deontológicos" (Valera, 2016: 116), es decir, “...la solución está en el periodismo de excelencia y en la labor esencial de garantizar una información veraz y contrastada" (Ortiz, 2015: 99). En este sentido, en los campos audiovisual y de la comunicación publicitaria, es necesario que las asociaciones hagan un esfuerzo para ser más transparentes ante sus públicos -instituciones, empresas, administraciones públicas, audiencias y sociedad en general-y para comunicar a la sociedad en qué consisten las actividades que desarrollan y porqué son importantes para el desarrollo económico, social y cultural.

Para alcanzar estos objetivos, hay que fortalecer el tejido asociativo y, aunque pueda parecer paradójico, las asociaciones profesionales del campo de la comunicación deben mejorar la gestión de su propia comunicación (Torner-Ventura, 2014), dedicar más recursos a la comunicación corporativa y desarrollar estrategias de comunicación más eficaces, potenciando también la cultura de la comunicación y la colaboración entre las diferentes asociaciones, y entre los ámbitos profesionales del periodismo, de la publicidad y del audiovisual. En este sentido, la creación de la Mesa Sectorial del Audiovisual Valenciano (MESAV) ha sido, a juicio de los encuestados, muy útil para poner en común los problemas y 
dificultades de diferentes ámbitos profesionales, todos ellos encuadrados dentro del marco general de la comunicación, en un momento histórico en que todos, sin diferencias significativas, han sufrido los efectos de una crisis de una gravedad sin precedentes. En el cuestionario realizado en 2019, se ha podido constatar cómo una vez se ha recuperado cierta normalidad en el campo de la comunicación, se ha producido una pérdida de la visión general que hizo posible la creación de la MESAV, cada vez más olvidada en la actualidad, como se constata en la última encuesta. La creación de la Academia Valenciana del Audiovisual (AVAV) constituye una iniciativa bien recibida entre los profesionales del audiovisual, sin duda necesaria, si bien se sitúa fuera de los campos de interés del periodismo y de la comunicación publicitaria que son ámbitos que parecen fundamentales para tener un visión completa del campo profesional de la comunicación.

Por otro lado, los profesionales constatan cómo la recuperación de los nuevos medios de comunicación públicos, de la Corporación Valenciana de Medios de Comunicación (CVMC), y su sociedad mercantil À Punt Mèdia, no puede verse como un proyecto finalizado. Por el contrario, es necesario aprender de la experiencia que ha supuesto el proceso de deterioro y cierre de RTVV. En este sentido, los profesionales de la comunicación ven imprescindible reactivar espacios comunes de encuentro para reivindicar la relevancia de la comunicación, de la diversidad audiovisual (Albornoz \& García-Leiva, 2017) y la calidad del sistema comunicativo valenciano (CECUV, 2015). En el nuevo contexto, À Punt Mèdia debe contribuir de forma muy significativa a la mejora de la reputación de las profesiones relacionadas con la comunicación, en tanto que se trata de la empresa audiovisual más importante de la Comunidad Valenciana (en tamaño, presupuesto, cobertura, etc.) que actúa como «motor», no sólo del sector audiovisual valenciano, sino también de las industrias culturales del territorio (López-Olano, 2018). El fracaso de RTVV lo fue en gran medida porque se descuidó la reputación de la institución que, a su vez, estaba estrechamente ligada al bajo nivel de engagement de sus empleados con la organización. Uno de los retos más importantes a los que se enfrenta À Punt es el desarrollo de una cultura corporativa, basada en conceptos como independencia, innovación, servicio público, gobernanza, transparencia, credibilidad y participación, que sólo se puede alcanzar con la complicidad de los empleados de la organización, mediante una comunicación interna de calidad (Marín-Pérez, 2019).

En este sentido, las universidades valencianas y, más concretamente, los espacios académicos vinculados a la docencia e investigación en comunicación - del periodismo, de la comunicación audiovisual y de la publicidad y las relaciones públicas- deben ser de gran ayuda para mejorar la imagen de los comunicadores y la reputación del sector comunicativo y audiovisual valenciano. La escasa participación del mundo académico en las encuestas de 2015, 2017 y 2019 es un síntoma de la falta de compromiso con la profesión y de la existencia de una distancia todavía muy importante entre el sistema educativo y el mundo profesional. No obstante, esta situación ha mejorado mucho en las últimas décadas. Desde la Asociación de Universidades con Titulaciones en Información y Comunicación (ATIC) y desde la Asociación Española de Investigación de la Comunicación (AEIC), cada vez son más frecuentes los estudios e investigaciones sobre la situación del mercado laboral, la evolución de los ámbitos profesionales de la comunicación, la emergencia de nuevos perfiles profesionales, el desarrollo de investigaciones aplicadas, etc. Sin lugar a dudas, la mejora de la reputación de las profesiones relacionadas con la comunicación está asimismo ligada a la reputación de la institución universitaria, que debe ser ejemplar en estos momentos. 
En el momento de finalizar la redacción del presente estudio, se declaraba el Estado de Alarma en España como consecuencia de la pandemia por el coronavirus COVID-19. Entre otras medidas, durante esta crisis el gobierno de España ha declarado a los medios de comunicación como servicios esenciales (BOE no 87, Anexos 11 y 13, 29/03/2020), lo que confirma la relevancia de la comunicación, en especial en situaciones tan complejas como esta. Y cabría añadir que cuando se habla de la relevancia de los medios, no sólo se refiere a la información, absolutamente necesaria en un estado de derecho, sino también al entretenimiento y a otros contenidos culturales y formativos, que han sido de gran ayuda a la ciudadanía para soportar mejor el tiempo de confinamiento al que se ha visto obligada la sociedad española. Apostar por la mejora de la reputación de las profesiones relacionadas con la comunicación es apostar por un futuro mejor, donde la comunicación está llamada a desarrollar un papel de máxima relevancia.

\section{Limitaciones del estudio}

La elección de la Comunidad Valenciana como ámbito geográfico para la realización del estudio se sustenta en el hecho de que se trata de un territorio de 5 millones de habitantes que aporta el 10\% del PIB español y que se ha convertido, en el periodo 2012-2018, en el único espacio europeo, con una cultura y lengua propias que ha sido privado de servicio público de RTV, una circunstancia excepcional, no sólo en Europa, sino también entre los países desarrollados de la OCDE. Por todo ello, la Comunidad Valenciana ofrece un campo de estudio de especial relevancia para analizar el escenario surgido tras la crisis del sistema comunicativo y audiovisual y para el análisis de acciones que puedan servir para mejorar la deteriorada reputación de las profesiones vinculadas a la comunicación, de aplicación extensible a otros territorios e, incluso, a nivel internacional.

Por otro lado, la introducción de nuevas preguntas en el cuestionario realizado en 2019, que no existían en los anteriores, ha obedecido a la necesidad de adaptar el estudio al nuevo contexto surgido tras la creación de À Punt Mèdia y la aparición de la Academia Valenciana del Audiovisual (AVAV).

\section{Agradecimientos}

Los autores del presente estudio quieren hacer constar su agradecimiento por la inestimable ayuda de Samuel Gil Soldevilla, Valentí Torner Ventura y Alicia Bruno Romero en la gestión de las encuestas y de los datos obtenidos.

\section{Financiación}

Este trabajo ha sido realizado con la ayuda del proyecto de investigación «Participación ciudadana y medios de comunicación públicos. Análisis de experiencias de co-creación audiovisual en España y en Europa (PARCICOM)» (código RT12018-093649-B-I00), bajo la dirección de Javier Marzal Felici, financiado por el Programa Estatal de l+D+i orientada a los Retos de la Sociedad, Ministerio de Ciencia, Innovación y Universidades, para el periodo 2019-2021. 


\section{Referencias bibliográficas}

Albornoz, L. A. \& García Leiva, M. T. (2017). Diversidad e industria audiovisual. El desafío cultural del siglo XXI. México: Fondo de Cultura Económica.

Alloza, A., Carreras, E. \& Carreras, A. J. (2013). Reputación corporativa. Madrid: LID Editorial.

Alvarez Villanueva, C. (2012). Manual de valoración de activos intangibles. La laguna, Tenerife: Cuadernos Artesanos Latina, Sociedad Latina de Comunicación Social. Disponible en https://cutt.ly/loC4ouS

Asociación de la Prensa de Madrid (2013). Informe de la Profesión Periodística 2013. Madrid: APM. Disponible en https:// cutt.ly/JoC83kO

Asociación de la Prensa de Madrid (2019). Informe de la Profesión Periodística 2019. Madrid: APM. Disponible en https:// cutt.ly/8oC4joP

Benavides, Juan; et al. (2015). La comunicación comercial en cambio permanente. Observatorio de la publicidad en España. Madrid: Asociación Española de Anunciantes. Disponible en https://cutt.ly/KoC4WB2

Bustamante, E. (2015). El servicio público en España: manual de las malas prácticas. En Marzal Felici, J.; Izquierdo Castillo, J. y Casero Ripollés, A. (eds.), La crisis de la televisión pública. El caso de RTVV y los retos de una nueva gobemanza. Barcelona, Bellaterra, Castellón y Valencia: Colección Aldea Global (UAB, UPF, UJI, UV), 23-37.

Campos Freire, F. (coord.) (2013). El futuro de la TV europea es híbrido, convergente y cada vez menos público. Revista Latina de Comunicación Social, 68. La Laguna (Tenerife): Universidad de La Laguna, pp. 89-118. Disponible en https:// cutt.ly/poC4370

Campos Freire, F. (coord.) (2016). Situación actual y tendencias de la radíotelevisión pública en Europa. Madrid: FORTA. Disponible en https://cutt.ly/hoC7sV1

Castells, M. (2009). Comunicación y poder. Madrid: Alianza.

CECUV (2015). Bases para la renovación del espacio comunicativo valenciano y la restitución del servicio público de radiotelevisión. Castellón de la Plana: Comisión de Expertos en Comunicación de las Universidades Valencianas, Universidades Valencianas. Disponible en https://cutt.ly/qoC7A8y

CIS (2013). Estudio no 2978. Barómetro de febrero 2013. Madrid: Centro de Investigaciones Sociológicas. Disponible en https://cutt.ly/doC7VgO

CIS (2020). Estudio no 3273. Estimación de voto de febrero 2020. Madrid: Centro de Investigaciones Sociológicas. Disponible en https://cutt.ly/eoC76Ln

Costa, J. (2003). Imagen Corporativa en el siglo XXI. Buenos Aires: La Crujía.

Duverger, M. (1971). Métodos de las ciencias sociales. Barcelona: Ariel.

EAVF (2014). Propuesta para una nueva Televisión Valenciana. Valencia: Empreses Audiovisuals Valencianes Federades. Disponible en https://cutt.ly/noC5lkY 
FAPAE (2015). Memoria FAPAE 2015. Madrid: Federación de Asociaciones de Productores Audiovisuales Españoles. Disponible en https://cutt.ly/ZoC6Blt

García-Santamaría, J. V. (2019). Las nuevas fronteras de la comunicación corporativa. Barcelona: UOC.

Galán Cubillo, E.; Gil-Soldevilla, S.; Soler-Campillo, M. \& Marzal-Felici, J. (2018). La estrategia transmedia de À Punt Mèdia y la nueva gobernanza. Hacia un nuevo modelo de RTV de proximidad. En: Galán Cubillo, E., Rodríguez Serrano, A. \& Marzal-Felici, J., Contenidos transmedia para la radiotelevisión de proximidad. Madrid: EUNSA, 125-150.

Hartley, J.; Wen, W. \& Li Henry, S. (2015). Creative Economy and Culture. Challenges, Changes and Futures for the Creative Industries. Londres: Sage. Disponible en http://dx.doi.org/10.4135/9781473911826

Helfat, C. E. \& Raubitschek, R. S. (2000). Product Sequencing: Co-Evolution of Knowledge, Capabilities and Products. Strategic Management Joumal, 21 (10/11), pp. 961-979. Disponible en https://dx.doi.org/10.2139/ssrn.237288 Hesmondhalgh, D. (2012). The Cultural Industries. Londres: Sage.

Juanatey-Boga, Ó., Martínez-Fernández, V. A. \& Rodríguez-Castro, M. (2018). La Europa de dos velocidades reflejada en la financiación del servicio audiovisual público: un análisis basado en los presupuestos. Revista Latina de Comunicación Social, 73, pp. 1054-1071. Disponible en https://doi/10.4185/RLCS-2018-1296

López-Rico, C. M. (2016a). El principio del fin de las televisiones públicas: la deriva de Canal 9. La Laguna, Tenerife: Cuadernos Artesanos de Comunicación. Disponible en https://cutt.ly/CoVqlqt

López-Rico, C. M. (2016b). La reapertura de radiotelevisión valenciana. De la manipulación al servicio público. Revista Mediterránea de Comunicación, 7(2), 243-257. Disponible en http://dx.doi.org/10.14198/MEDCOM2016.7.2.15

López-Olano, C. (2018). RTVV: Paradigma de la triple crisis de las televisiones públicas. Valencia: Tirant Lo Blanch.

Marín-Pérez, B. (2019). Convertir a los colaboradores en embajadores de la marca: plan de comunicación interna para la televisión autonómica valenciana À punt. El profesional de la información, v. 28, n. 5, e280515. Disponible en https://doi. org/10.3145/epi.2019.sep.15

Marzal-Felici, Javier (ed.) (2015). Las televisiones públicas autonómicas del siglo XXI. Nuevos escenarios tras el cierre de RTVV. Barcelona, Bellaterra, Castellón y Valencia: Colección Aldea Global. (UAB, UPF, UJI, UV).

Marzal-Felici, J. \& Soler-Campillo, M. (2017). El impacto del cierre de RTVV en la prensa española y valenciana. Algunas reflexiones sobre la inmadurez del sistema comunicativo valenciano. En: Marzal-Felici, J.; López-Rabadán, P. \& Izquierdo-Castillo, J. (eds.) (2017). Los medios de comunicación públicos de proximidad en Europa. RTVV y la crisis de las televisiones públicas. Valencia: Tirant Humanidades, pp. 273-303.

Matas, Antonio (2018). Diseño del formato de escalas tipo Likert: un estado de la cuestión. Revista Electrónica de Investigación Educativa, 20(1), 38-47. Disponible en https://doi.org/10.24320/redie.2018.20.1.1347

MESAV (2014). Hacia un nuevo espacio audiovisual. Valencia: Mesa Sectorial del Audiovisual Valenciano. Disponible en https://cutt.ly/UoVwIME

Miége, B. (2004). L'information-communication objet de connaissance. Bruselas: De Boeck-lNA. 
Newman, N., Fletcher, R., Kalogeropoulos, A. \& Nielsen, K. N. (2019). Reuters lnstitute Digital News Report 2019. Oxford: Reuters lnstitute \& University of Oxford. Disponible en https://cutt.ly/4oVeq4A

Ortiz de Guinea Ayala, Y. (2015). El periodismo y la reputación de los periodistas en tiempos de crisis. Index comunicación, 5 (2), pp. 83-100. Disponible en https://cutt.ly/6oVelKo

PAV (2019). Informe diagnóstico del sector audiovisual de la Comunidad Valenciana. Valencia: Productores Audiovisuales Valencianos. Generalitat Valenciana, Productores Audiovisuales Valencianos y Equipo Humano Consultores. Disponible en https://cutt.ly/VoVeI5j

Piñeiro-Aguilar, E. (2015). Observación participante: una introducción. Revista San Gregario, Número Especial 1, Metodología de la investigación, pp. 80-89. Disponible en https://cutt.ly/PoVrpAn

Prado, E. (2015). Indispensabilidad de la televisión pública en el universo convergente. En: Marzal Felici, Javier; Izquierdo Castillo, Jessica; Casero Ripollés, Andreu (eds.), La crisis de la televisión pública. El caso de RTW y los retos de una nueva gobemanza. Barcelona, Bellaterra, Castellón y Valencia: Colección Aldea Global (UAB, UPF, UJI, UV).

Sánchez-Revilla, M. Á. et al. (2014). Estudio lnfoadex de la inversión publicitaria en España 2014. Madrid: lnfoadex. Disponible en https://cutt.ly/qoVrxHj

Soler-Campillo, M. \& Marzal-Felici, J. (2015). La relevancia estratégica de RTVV en el sistema comunicativo valenciano y para el desarrollo económico, social y cultural de la Comunidad Valenciana. En: Marzal-Felici, J.; Izquierdo-Castillo, J. \& Casero-Ripollés, A. (eds.), La crisis de la televisión pública. El caso de RTVVy los retos de una nueva gobernanza. Barcelona, Bellaterra, Castellón y Valencia: Colección Aldea Global (UAB, UPF, UJI, UV).

Soler-Campillo, M., Galán-Cubillo, E. \& Marzal-Felici, J. (2019). La creación de A Punt Media (2013-19) como nuevo espacio público de comunicación. Revista Latina de Comunicación Social, 74, pp. 1801-1817. http://www.revistalatinacs. org/074paper/1411/94es.html

Teece, D. J., Pisana, G. \& Shuen, A. (1997). Dynamic Capabillties and Strategic Management. Strategic Management Joumal, 18 (7), pp. 509-533. Disponible en https://doi.org/10.1002/(SICI)1097-0266(199708)18:7<509::AID-SMJ882>3.0.CO;2-Z Torner-Ventura, V. (2014). Gestión de la comunicación en las asociaciones profesionales de comunicación de la Comunidad Valenciana. Fórum de Recerca, 19/2014, pp.607-624. Disponible en http://dx.doi.org/10.6035/ForumRecerca.2014.19.38 UNESCO (2013). Informe sobre la economía creativa. Ampliar los cauces de desarrollo local. Paris: Programa de las Naciones Unidas para el Desarrollo. Disponible en https://cutt.ly/MoVteTO

UPV (2015). Un model pera la comunicació audiovisual valenciana. Valencia: Unió de Periodistes Valencians. Disponible en https://cutt.ly/2oVtsxC

Valera, S. J. (2016). Quiebra total del sistema de medios español: requisitos para sobrevivir en el nuevo ecosistema. Cuadernos de Periodistas, 32, Asociación de la Prensa de Madrid, pp. 101-116. Disponible en https://cutt.ly/yoVtmcJ

Villafañe, J. (2004). La buena reputación. Claves del valor intangible de las empresas. Madrid: Ediciones Pirámide.

Villafañe, J. (2013). La buena empresa. Propuesta para una teoría de la reputación corporativa. Madrid: Pearson. 
Vogel, Harold L. (2004). La industria de la cultura y el ocio. Un análisis económico. Madrid: Fundación Autor.

Zallo, R. (2011). Estructuras de la comunicación y la cultura: políticas para la era digital. Barcelona: Gedisa.

Zallo, R. (2015). Razones e implicaciones del cíerre de Radiotelevisión Valenciana: pensar en el futuro. En Marzal Felici, Javier; Izquierdo Castillo, Jessica; Casero Ripollés, Andreu (eds.) (eds.), La crisis de la televisión pública. El caso de RTVV y los retos de una nueva gobernanza, Barcelona, Bellaterra, Castellón, Valencia: Universitat Pompeu Fabra, Universitat Autónoma de Barcelona, Universitat Jaume 1, Universitat de Valencia. Collecció Aldea Global.

Zerfass, A.; Vercic, D.; Verhoeven, P.; Moreno, Á.; Tench, R. (2019). European Communication Monitor 2019. Exploring trust in the profession, transparency, artificial inte/ligence amd new content strategies. Results of a survey in 46 countries. Brussels: EUPRERNEACD, Quadriga Media Berlin. Disponible en https://cutt.ly/HoVu3vW 